\title{
$308 \mathrm{~nm}$ 激光作用下的 $\mathrm{CH}_{3} \mathrm{OH} 、 \mathrm{C}_{2} \mathrm{H}_{5} \mathrm{OH}$ 多光子电离过程
}

\author{
关一夫韩景诚
}

刘厚祥 李书涛 吴存恺

(中成科学院安僧光学棈密机械研究所,合肥)

为了搞清激光辐照酒以加速其醇化过程的机理, 我们利用 $\mathrm{XeCl}$ 准分子激光进行了 $\mathrm{CH}_{3}$ $\mathrm{OH}$ 和 $\mathrm{C}_{2} \mathrm{H}_{5} \mathrm{OH}$ 的多光子电离质谱实验, 初步了解了其中的一些动力学过程.

\section{一、实验装置和实验结果}

图 1 是分子多光子电离质谱实验的示意图. 在分子束真空室中, 聚焦后的激光束与分子

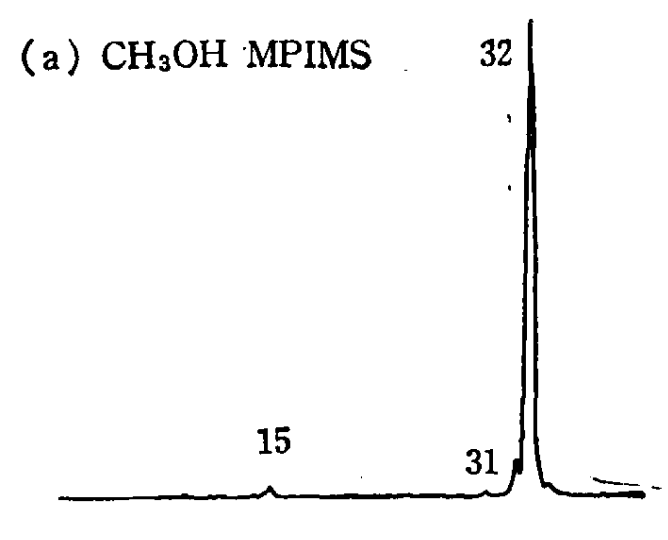

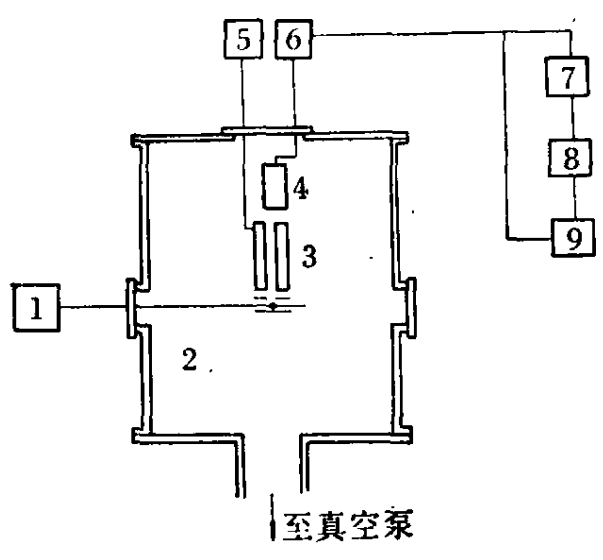

图 1 分子多光子电离质谱实验的示意图

1. $\mathrm{XeCl}$ 准分子湤光器; 2. 分子束 真空空；3. 四极杆探头；4. 电子倍增 器；5. 质息扫掉控制；6. 前置放大器; 7. Boxcar 信号平均器; 8. X-Y 记录 仪：9. 示波器

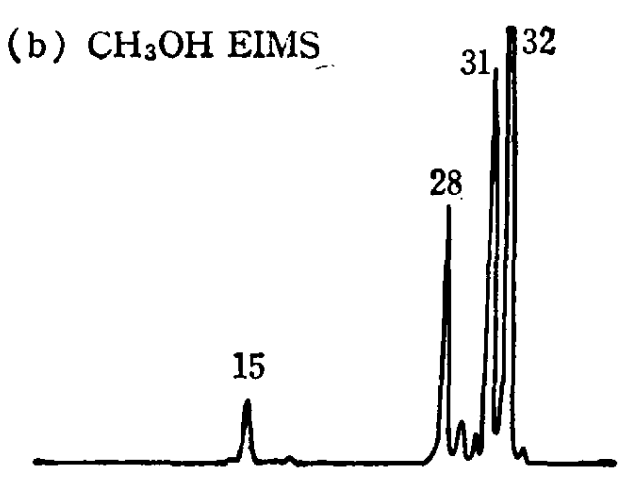

图 2

（a） $\mathrm{CH}_{3} \mathrm{OH}$ 多光子电离质谱; (b) $\mathrm{C}_{2} \mathrm{H}_{3} \mathrm{OH}$ 电子表击电离质棬

束垂直相交，从而使分子吸收多个光子而成为离子.由激光产生的离子在加速电场排斥下进人 四极杆电场内, 随着四极电场扫描电压的渐变各种不同质量的离子依次地被电子倍增器所接 收、放大, 其信号经 Boxcar 信号平均器平均后由 $\mathrm{X}-\mathrm{Y}$ 记录仪绘出多光子电离质谱. 实验装 
置的详细描述可见文献 [1].

在图 2 的 $\mathrm{CH}_{3} \mathrm{OH}$ 多光子电离质谱中有三个离子峰, 它们是 $\mathrm{CH}_{3}^{+}(m / c=15), \mathrm{CH}_{2} \mathrm{OH}^{+}$ $(m / e=31)$ 和 $\mathrm{CH}_{3} \mathrm{OH}^{+}(m / e=32)$. 实验测得的三种离子的离子强度随着样品浓度变化 的指数因子 $K_{P}$ 分别是 $2.45 \pm 0.43,2.46 \pm 0.28$ 和 $1.29 \pm 0.06$. 在最大光强下离子的相对强度 分别是 $6.4 \%, 2.4 \%$ 和 $91.2 \%$. 由于离子强度相差悬殊, 只测量了 $\mathrm{CH}_{3} \mathrm{OH}^{+}$的强度随激光光 强变化的指数因子 $K_{I}=3.91 \pm 0.21 . K_{P}$ 和 $K_{I}$ 的测量结果分别示于图 4、图 5 中.

(b) $\mathrm{C}_{2} \mathrm{H}_{5} \mathrm{OH}$ EIMS

(a) $\mathrm{C}_{2} \mathrm{H}_{5} \mathrm{OH}$ MPIMS
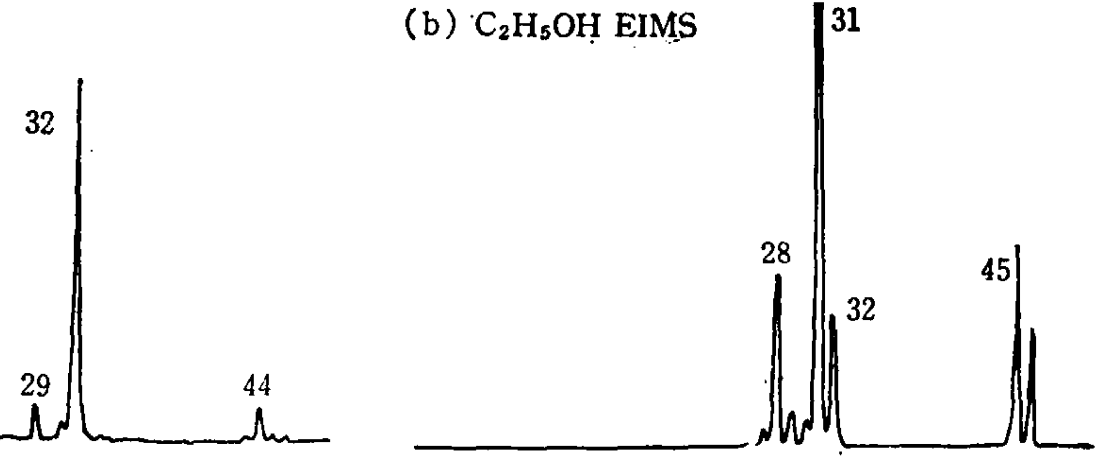

图 3

(a) $\mathrm{C}_{2} \mathrm{H}_{3} \mathrm{OH}$ 多光子电离质谱; (b) $\mathrm{C}_{2} \mathrm{H}_{9}, \mathrm{OH}$ 电子轰击电离质谱
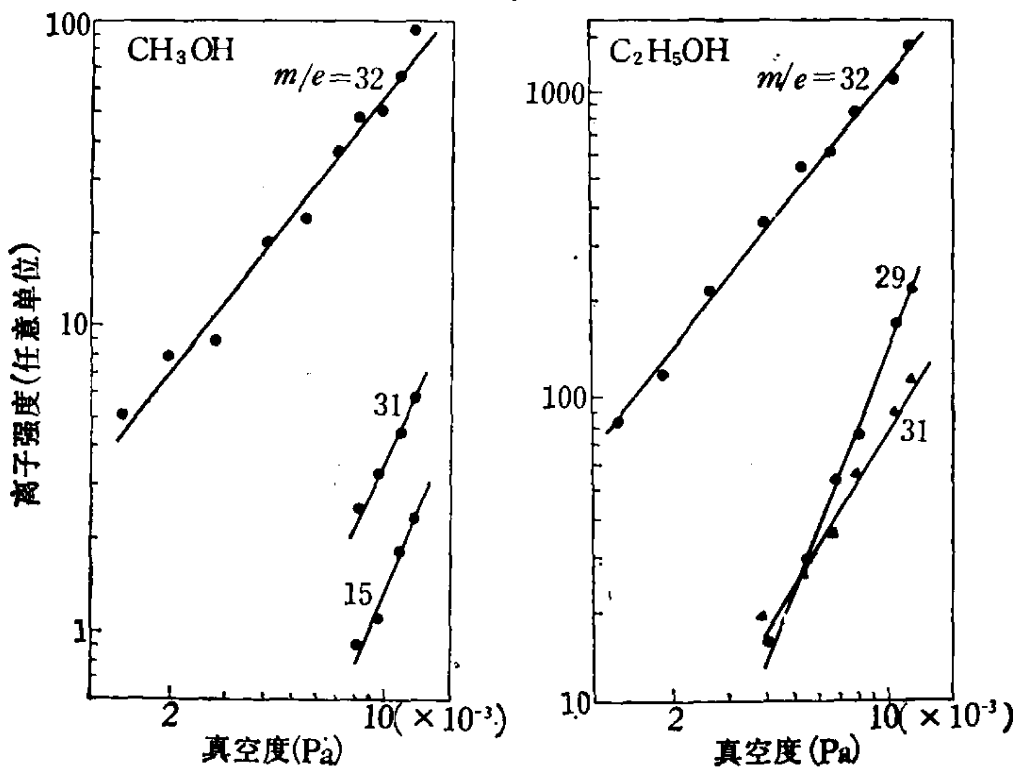

图 4 离子强度随样品浓度变化的曲线

在图 3 的 $\mathrm{C}_{2} \mathrm{H}_{5} \mathrm{OH}$ 多光子电离质谱中出现的离子分别标定为 $\mathrm{C}_{2} \mathrm{H}_{5}^{+}(m / e=29), \mathrm{CH}_{2}-$ $\mathrm{OH}^{+}(m / e=31), \mathrm{CH}_{3} \mathrm{OH}^{+}(m / e=32), \mathrm{C}_{2} \mathrm{H}_{2} \mathrm{OH}^{+}(m / e=43), \mathrm{C}_{2} \mathrm{H}_{3} \mathrm{OH}^{+}(m / e=44)$, $\mathrm{C}_{2} \mathrm{H}_{4} \mathrm{OH}^{+}(m / e=45), \mathrm{C}_{2} \mathrm{H}_{5} \mathrm{OH}^{+}(m / e=46)$. 离子峰相对 强度 是 $74.2 \% \quad(m / e=32)$, $12 \%(m / e=29), 6.1 \%(m / e=31), 4.1 \%(m / e=44)$, 其余几种离子的相对强度均在 $2 \%$ 以下. 实验测得的光强指数因子是 $K_{I}(m / e=29)=2.26 \pm 0.35, K_{I}(m / e=31)-2.02$ $\pm 0.19, K_{l}(m / e=32)=3.47 \pm 0.27$, 气量指数因子分别是 $K_{P}(m / e=29)=2.50 \pm 0.06$, 

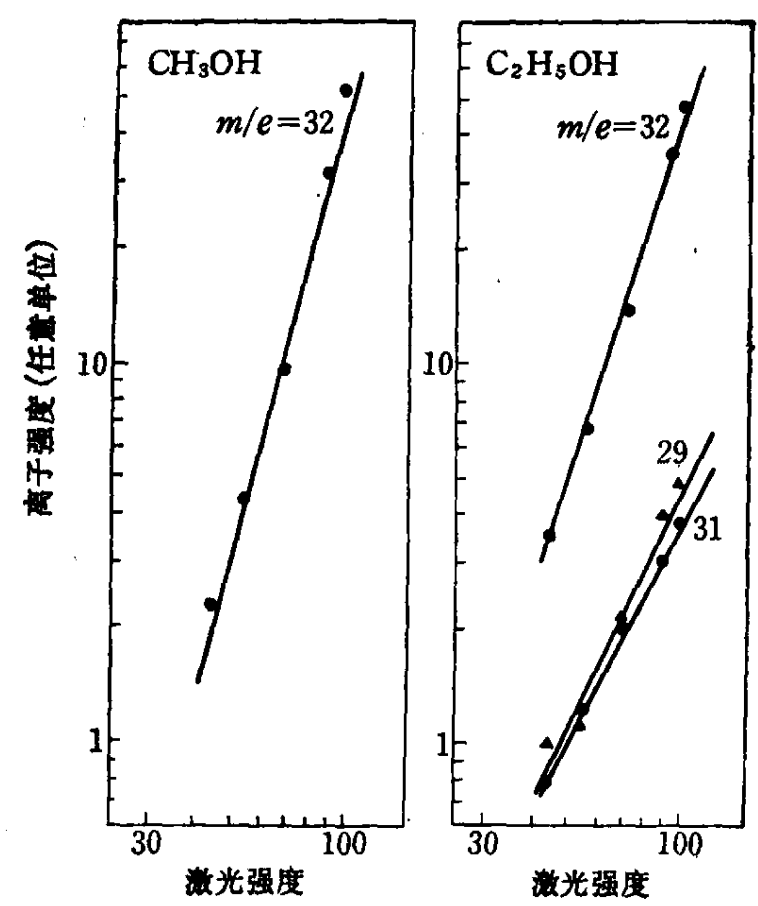

图 5 离子强度随激光强度变化的曲线

$K_{P}(m / e-31)=1.68 \pm 0.09, K_{P}(m / e=32)=1.28 \pm 0.04$. 测量结果参见图 4 和图 5.

\section{二、分析和讨论}

根据质谱中离子的变化规律以及分子、离子的能级结构, 我们对 $\mathrm{CH}_{3} \mathrm{OH}$ 和 $\mathrm{C}_{2} \mathrm{H}_{5} \mathrm{OH}$ 多 光子电离过程做如下分析.

在 $\mathrm{CH}_{3} \mathrm{OH}$ 多光子电离过程中, 母体分子 $\mathrm{CH}_{3} \mathrm{OH}$ 吸收四个光子 $\left(K_{I} \doteq 4\right)$ 后直接生成 母体离子 $\mathrm{CH}_{3} \mathrm{OH}^{+}$, 这显然是一个单分子过程 $\left(K_{P}=1\right)$. 由于离子在排斥场作用下进人四 极杆电场的运动方向与分子束中分子的运动方向相垂直, 故 $\mathrm{CH}_{3} \mathrm{OH}^{+}$有机会与中性 分子发 生碰撞而离解成为 $\mathrm{CH}_{3}^{+}$和 $\mathrm{CH}_{2} \mathrm{OH}^{+}$.

$$
\begin{aligned}
\mathrm{CH}_{3} \mathrm{OH}+4 h v & \longrightarrow \mathrm{CH}_{3} \mathrm{OH}^{+} \\
\mathrm{CH}_{3} \mathrm{OH}^{+}+\mathrm{CH}_{3} \mathrm{OH} & \longrightarrow \mathrm{CH}_{3}^{+}+\mathrm{OH}+\mathrm{CH}_{3} \mathrm{OH} \\
& \longrightarrow \mathrm{CH}_{2} \mathrm{OH}^{+}+\mathrm{H}+\mathrm{CH}_{3} \mathrm{OH}
\end{aligned}
$$

图 2 中 $\mathrm{CH}_{3} \mathrm{OH}$ 电子轰击质谱和多光子电离质谱的明显差异起因于两个电离过程的不 同. 在电子戔击电离过程中, 轰击电子的能量分布在较大范围内, 这可使离子分布在不同的离 子能级上,造成了质谱的多峰结构. 在多光子电离过程中, 激光的选择性激发特性能将离子布 居到特定的能级上，从而形成了有别于电子轰击质谱中谱分布的多光子电离质谱. 根据实验 结果可以推断在 $308 \mathrm{~nm}$ 激光作用下四光子形成的 $\mathrm{CH}_{3} \mathrm{OH}^{+}$是一个较稳定的离子.

按照一般分子键断裂的情况, 可以预计在 $\mathrm{C}_{2} \mathrm{H}_{5} \mathrm{OH}$ 多光子电离质谱中会观察到 $m / e=$ $15,17,29,31$ 的离子峰出现. 并且按照分子离子碎裂的有关规律,如在含有杂原子的有机分 子中, 与杂原子相邻的 $\mathrm{C}-\mathrm{C}$ 键最易发生断裂过程并将正电荷留在含杂原子的碎片上; 烷烃分 子 $\mathrm{C}_{n} \mathrm{H}_{2 \mathrm{n}+1}$ 离子稳定性较强等, 可以预计 $m / e=15,29,31$ 的离子会有较大的强度 ${ }^{[2,33}$. 


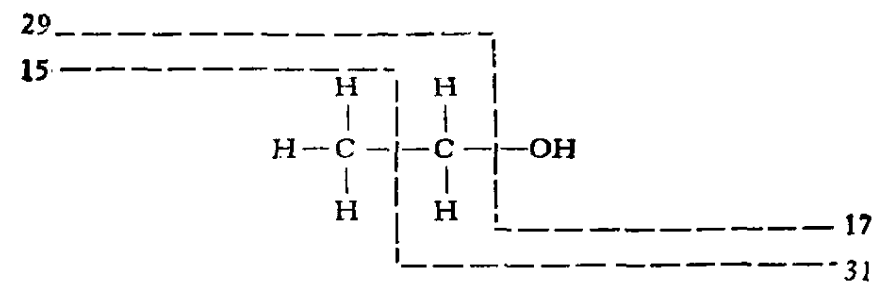

实验得到的 $\mathrm{C}_{2} \mathrm{H}_{5} \mathrm{OH}$ 多光子电离质谱与预计结果出入很大, $\mathrm{CH}_{3}^{+}$和 $\mathrm{OH}^{+}$根本不存在, $\mathrm{C}_{2} \mathrm{H}_{5}^{+}$和 $\mathrm{CH}_{2} \mathrm{OH}^{+}$强度分别只有 $12 \%$ 和 $6.1 \%$, 而预计之外的 $\mathrm{CH}_{3} \mathrm{OH}^{+}(m / e=32)$ 却成了 质谱中的主要成分. 质谱理论业已指出, 由一种离子生成另外一种离子不仅可以通过一些分子 键的断裂来实现 (图 3(b) 中 $\mathrm{CH}_{2} \mathrm{OH}^{+}$的形成), 而且还可以通过分子中原子的重排过程实现 (图 3(b) 中 $m / e=28$ 的离子, 它是 $\mathrm{C}_{2} \mathrm{H}_{5} \mathrm{OH}^{+}$的脱水过程形成的 $\mathrm{C}_{2} \mathrm{H}_{4}^{+}$). 在 $\mathrm{C}_{2} \mathrm{H}_{5} \mathrm{OH}$ 多光 子电离过程中, $\mathrm{CH}_{3} \mathrm{OH}^{+}$的形成不能用上面的二个过程解释, 它是一个有待于查明的复杂的 复合过程。

在 $\mathrm{C}_{2} \mathrm{H}_{5} \mathrm{OH}^{+}$附近的一组离子峰, 以 $\mathrm{C}_{2} \mathrm{H}_{3} \mathrm{OH}^{+}$居多. 由 $\mathrm{C}_{2} \mathrm{H}_{5} \mathrm{OH}^{+}$到 $\mathrm{C}_{2} \mathrm{H}_{3} \mathrm{OH}^{+}$满足 于重排条件, 并由二者的电离电位 (I. $\mathrm{P}\left(\mathrm{C}_{2} \mathrm{H}_{3} \mathrm{OH}\right)=8.85 \mathrm{eV}$, I. $\mathrm{P}\left(\mathrm{C}_{2} \mathrm{H}_{5} \mathrm{OH}\right)=10.50 \mathrm{eV}$ ) 可 知, 这一重排过程是 McLaffety 重排 ${ }^{[3]}$, 其过程是当 $\mathrm{C}_{2} \mathrm{H}_{5} \mathrm{OH}^{+}$释放出二个氢原子后生成乙烯 醇离子,由于这个离子本身不稳定, 它经过烯醇一酮互变异构过程生成乙醛离子, 而后者具有一 定的稳定性.

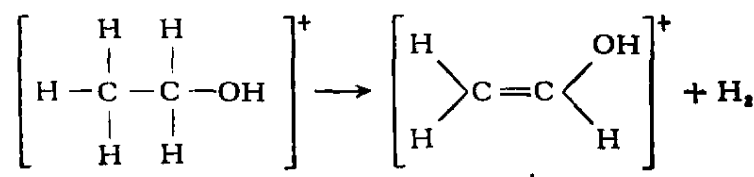

$$
\begin{aligned}
& {\left[\begin{array}{c}
\mathrm{H} \\
\mathrm{i}-\mathrm{C}-\mathrm{C} \\
\mathrm{H}
\end{array}\right]_{\mathrm{H}}^{\mathrm{O}}}
\end{aligned}
$$

实验结果表明, 在气相条件下, $\mathrm{CH}_{3} \mathrm{OH}$ 在 $\mathrm{XeCl}$ 准分子激光作用下生成母体离子 $\mathrm{CH}_{3} \mathrm{OH}^{+}$, 而 $\mathrm{C}_{2} \mathrm{H}_{5} \mathrm{OH}$ 在相同条件下的多光子电离过程也产生了大量的 $\mathrm{CH}_{3} \mathrm{OH}^{+}$. 在用 $\mathrm{XeCl}$ 准分子 激光辐射酒的过程中应适当地考虑到这一点.

\section{参考文献}

[1] 关一夫、韩景诚、刘颂豪, 光学学报, 6(1986), 2: 162-168.

[2] Silverstein, R. M., Bassler, G. C. and Morril, T. C., Spectrometric Identification of Organic Compounds, John Wiley, New York, 1974, 4-15.

[3] 洪山海, 光谱解析法在有机化学中的应用, 科学出版社, 北京, 1980, 251-274. 